\title{
Randomised, controlled trial of nasal continuous positive airway pressure in the extubation of infants weighing 600 to $1250 \mathrm{~g}$
}

\author{
Peter Davis, Robert Jankov, Lex Doyle, Philip Henschke
}

\begin{abstract}
Aim-To determine whether extubation to nasal continuous airway pressure (NCPAP) results in a greater proportion of infants remaining free of additional ventilatory support for one week after extubation compared with those extubated directly to headbox oxygen.
\end{abstract}

Methods-A randomised, controlled, clinical trial was conducted at the neonatal intensive care unit of the Royal Women's Hospital, Melbourne, of infants with birthweights between 600 and $1250 \mathrm{~g}$, ventilated via an endotracheal tube for more than 12 hours, requiring less than $50 \%$ oxygen, a ventilator rate $\leqslant 20 /$ minute, considered by the clinical management team to be ready for extubation. Infants were randomly allocated either to NCPAP or to oxygen administered via a headbox. Success was defined by no requirement for additional ventilatory support over the week following extubation. Failure criteria were (i) apnoea; (ii) absolute increase in oxygen requirement greater than $15 \%$ above that required before extubation; or (iii) respiratory acidosis $(\mathrm{pH}<7.25$ with pCO $_{2}>6.67 \mathrm{kPa}$ ).

Results-Thirty one of $47(66 \%)$ infants were successfully extubated to NCPAP compared with 18 of $45(40 \%)$ for headbox oxygen. The increase in failure rate in the headbox group was due primarily to increased oxygen requirements in this group. Of the 27 who failed headbox oxygen, 26 were given a trial of NCPAP and 13 did not require endotracheal reintubation. There was no significant difference between the groups in the total number of days of assisted ventilation or the duration of inpatient stay.

Conclusions-NCPAP applied prophylactically after endotracheal extubation reduces the incidence of adverse clinical events that lead to failure of extubation in the seven days after extubation. This reduction is clinically important. The benefits of NCPAP do not seem to be associated with an increased incidence of unwanted side effects.

(Arch Dis Child Fetal Neonatal Ed 1998;79:F54-F57)

Keywords: ventilation; nasal continuous positive airway pressure; extubation

Preterm infants frequently receive intermittent positive pressure ventilation (IPPV) via an endotracheal tube in the days after birth. Although potentially lifesaving, IPPV is associated with clinically significant morbidity, including chronic lung disease, damage to the upper airway, and systemic and pulmonary infection. Early removal of the endotracheal tube is often attempted to prevent these problems. Failure to make the transition to spontaneous, unassisted ventilation following extubation is relatively common. Factors contributing to failure include upper airway instability, alveolar atelectasis, and poor respiratory drive. Continuous positive airway pressure was first used in neonates in 1971 for the treatment of respiratory distress syndrome. ${ }^{1}$ Potential benefits in the care of infants immediately following endotracheal extubation include prevention of atelectasis ${ }^{2}$ and improved oxygenation, ${ }^{3}$ a decrease in the work of breathing ${ }^{4}$ and improved breathing patterns, with a decrease in the frequency and severity of episodes of apnoea. ${ }^{3}$

Previous studies using different levels and methods of nasal continuous positive airway pressure (NCPAP) administration have yielded conflicting results with respect to efficacy of this mode of support. ${ }^{25-9}$ With the exception of successful extubation and chronic lung disease (CLD),${ }^{578}$ the impact of method of after extubation care on other important neonatal outcomes has not been evaluated. We conducted a prospective, randomised trial to determine whether NCPAP, administered via a single, short nasal prong, resulted in a greater proportion of infants being successfully extubated, without unwanted side effects, compared with management in headbox oxygen.

\section{Methods}

All babies (inborn and outborn) admitted to the neonatal intensive care unit at the Royal Women's Hospital, Melbourne, were considered for participation in this trial. Eligibility criteria included (i) birthweight between 600 and $1250 \mathrm{~g}$, (ii) endotracheal intubation for at least 12 hours, and (iii) stable or improving respiratory status with a ventilator rate less than or equal to $20 /$ minute and an inspired oxygen concentration of less than $50 \%$. Informed parental consent was obtained before randomisation (sequentially numbered, sealed, opaque envelopes using a random number table). Infants were stratified by gender, birthweight (600-800 and 801-1250 g) and postnatal age (less than or equal to 14 days and greater than 14 days) for a total of eight strata. Variable block sizes were used within each stratum. 
All infants received a loading dose of theophylline and were extubated from low rate ventilation rather than endotracheal CPAP or high frequency oscillation (HFO). The sealed envelope was opened immediately before extubation and infants were allocated to receive either NCPAP or headbox oxygen. NCPAP was administered via a size 2.5 or 3.0 Portex tube (Portex Ltd. Hyth, Kent, England) inserted $2.5 \mathrm{~cm}$ into one nostril and connected to a Bear Cub Ventilator (Bear Medical Systems Inc. Riverside, CA, USA). Humidified oxygen was provided at the same concentration as that required before extubation and CPAP level set at $7 \mathrm{~cm}$ water initially. The CPAP level was weaned according to the infant's status to a level of $5 \mathrm{~cm}$ water before extubation to headbox oxygen. Infants randomised to headbox were initially placed in an ambient oxygen concentration $5 \%$ greater than that required before extubation. Both groups had inspired oxygen concentrations adjusted to maintain oxygen saturation levels between 90 and 95\%. Capillary or arterial blood gases were performed six hours after extubation and then at least daily for the week after extubation.

Successful extubation was determined over the seven days following removal of the endotracheal tube. An infant was deemed to have failed if any of the following occurred:

(1) apnoea: minor-more than six episodes requiring physical stimulation over six hours, or major-one episode requiring bag and mask ventilation, or;

(2) an absolute increase in oxygen concentration of greater than $15 \%$ above that required immediately prior to extubation, or;

(3) respiratory acidosis $-\mathrm{pH}<7.25$ with $\mathrm{pCO}_{2}$ $>6.67 \mathrm{kPa}$.

Infants who reached the failure criteria could be given a trial on the other mode of treatment at the attending physician's discretion before reintubation of the trachea. These infants were classified as "failures" in the primary analysis. Thus infants were classified as being successfully extubated if they did not meet the failure criteria and therefore did not require treatment with either the alternative strategy or endotracheal reintubation.

We recorded the need for reintubation following initial extubation; the overall number of days on assisted ventilation; and the duration of stay at the study centre. Weight gain over the week following extubation and over the period from extubation to discharge from hospital was

Table 1 Patient characteristics

\begin{tabular}{lll}
\hline & NCPAP $(n=47)$ & Headbox $(n=45)$ \\
\hline Birthweight $(\mathrm{g})^{\star}$ & $960(182)$ & $927(157)$ \\
Gestational age (weeks) ${ }^{\star}$ & $27(2)$ & $27(2)$ \\
Age at extubation (days) $\dagger$ & $3(1-5)$ & $4(1.5-7)$ \\
Inborn/outborn & $46 / 1$ & $44 / 1$ \\
Male/female & $30 / 17$ & $29 / 16$ \\
Single/multiple & $38 / 9$ & $32 / 13$ \\
Maternal steroids & $37(79 \%)$ & $38(84 \%)$ \\
Surfactant & $36(77 \%)$ & $38(84 \%)$ \\
Inspired oxygen concentration at extubation $\dagger$ & $21(21-23)$ & $21(21-23.5)$ \\
Rate at extubation (breaths/minute) $\dagger$ & $15(10-15)$ & $15(10-15)$ \\
Mean airway pressure at extubation (cm $\left.\mathrm{H}_{2} \mathrm{O}\right)^{\star}$ & $5.6(0.9)$ & $5.7(1.3)$ \\
ॠmean (standard deviation); tmedian (interquartile range)
\end{tabular}

*mean (standard deviation); †median (interquartile range) measured. Feeding intolerance was defined as the presence of a four hourly gastric aspirate greater than $25 \%$ of the feed volume or containing bile during the week after extubation. Sepsis was diagnosed on the basis of positive culture of blood, cerebrospinal fluid, or bladder tap urine. Suspected sepsis was defined as a culture negative episode, treated with at least five days of antibiotics in conjunction with an immature to total neutrophil ratio of greater than 0.15. Cranial ultrasound examinations were performed within the first three days of life, days seven and 28, and then monthly until discharge; cerebroventricular haemorrhage was classified according to Papile. ${ }^{10}$ Infants were examined every two weeks by an experienced ophthalmologist and retinopathy of prematurity (ROP) classified according to ICD code. ${ }^{11}$ Chronic lung disease was determined by supplemental oxygen requirement at (1) 28 days of age and (2) 36 weeks of corrected age.

Sample size calculations were based on a retrospective chart review which found a $50 \%$ failure rate in a group of infants in this birthweight range extubated to headbox oxygen. A reduction of this failure rate to $25 \%$ was the clinically important improvement chosen to establish NCPAP as the preferred method of treatment, given its greater expense and invasiveness. A sample size of 130 was calculated to provide a power of $80 \%$ to detect a reduction in failure rate from $50 \%$ to $25 \%$ with two tailed $\alpha$ of less than 0.05 . Interim analyses were planned at 50 and 90 patients. According to the O'Brien-Fleming procedure, ${ }^{12}$ the $\mathrm{p}$ values to stop the trial were $\mathrm{p}<0.0006$ after a third of patients had been entered and $\mathrm{p}<0.015$ after two thirds. $\chi^{2}$ analysis was used for comparison of failure rates and other categorical patient variables. The two tailed Student's $t$ test and the Mann-Whitney $\mathrm{U}$ test were used for continuous data, where appropriate.

The study was approved by the research and ethics committees of the Royal Women's Hospital, Melbourne, Victoria, Australia.

\section{Results}

From 13 March 1995 through to 19 August 1996, 128 infants with birthweights between 600 and $1250 \mathrm{~g}$ were admitted to the neonatal intensive care unit and ventilated for longer than 12 hours. Sixteen infants died before extubation and consent was not obtained in an additional 16 cases. Three patients were overlooked for randomisation, and one patient was excluded by physician request because of a pulmonary haemorrhage several days before extubation. Ninety two infants were therefore randomised to receive either NCPAP or headbox oxygen.

There were no significant differences in the baseline demographic variables known to influence neonatal outcome (table 1). Babies reached minimal levels of ventilatory support and were extubated at a median of 3 and 4 days of age (table 1).

Table 2 shows that 31 of 47 infants were successfully extubated to NCPAP compared 
Table 2 Results expressed as number (\%)

\begin{tabular}{|c|c|c|c|}
\hline & $\operatorname{NCPAP}(n=47)$ & Headbox $(n=45)$ & \\
\hline \multicolumn{4}{|l|}{ Success: } \\
\hline All & $31(66)$ & $18(40)$ & $\mathrm{p}=0.013$ \\
\hline$\geqslant 800 \mathrm{~g}$ & $1 / 11(9)$ & $2 / 10(20)$ & \\
\hline$>800 \mathrm{~g}$ & $30 / 36(83)$ & $16 / 35(46)$ & $\mathrm{p}=0.0009$ \\
\hline Death & $2(4)$ & $2(4)$ & \\
\hline Death or in $\mathrm{O}_{2}$ at 28 days & $31(66)$ & $23(51)$ & \\
\hline Death or in $\mathrm{O}_{2}$ at 36 weeks & $15(32)$ & $14(31)$ & \\
\hline Grade 3 or 4 IVH & $5(11)$ & $4(9)$ & \\
\hline PVL & $4 / 43(9)$ & $2 / 44(5)$ & \\
\hline Any ROP & $11 / 45(24)$ & $12 / 44(27)$ & \\
\hline Grade 3,4 , or 5 ROP & $4 / 45(9)$ & $3 / 44(7)$ & \\
\hline Sepsis & $18(38)$ & $13(29)$ & \\
\hline Suspected sepsis & $23(49)$ & $14(31)$ & \\
\hline Feeding intolerance ${ }^{\star}$ & $23(49)$ & $20(44)$ & \\
\hline Weight gain ${ }^{\star}+$ & $3 \mathrm{~g} /$ day $(-8,10)$ & $0 \mathrm{~g} /$ day $(-7,8.5)$ & \\
\hline Weight gain (extubation to discharge) $\dagger$ & $20 \mathrm{~g} /$ day $(6,26)$ & $20 \mathrm{~g} /$ day $(16,26)$ & \\
\hline Endotracheal reintubation ${ }^{\star}$ & $34(16)$ & $31(14)$ & \\
\hline Days on IPPV/HFO/NCPAP† & $28(13-39)$ & $31(7-44)$ & \\
\hline Days on IPPV/HFO $†$ & $5(2-15)$ & $6(2-19)$ & \\
\hline Days at level 3 centre $\dagger$ & $63(40-79)$ & $70(41-82)$ & \\
\hline
\end{tabular}

with 18 of 45 for headbox oxygen ( $p=0.013$ ). Of the causes of failure, increased oxygen requirements contributed most to the difference between the two groups (table 3). Analysis by birthweight strata showed a significant benefit for infants extubated to NCPAP only in those with birthweights greater than $800 \mathrm{~g}$; the number of infants less than $800 \mathrm{~g}$ was insufficient to establish a significant difference between the groups (table 2). Only six infants were extubated after 14 days of age (two CPAP, four headbox). In the study protocol crossover was allowed in both directions, but in practice only infants failing headbox oxygen were tried on the alternative treatment. Thirteen of the 26 headbox infants tried on "rescue" NCPAP remained extubated for seven days. There was no significant difference in the length of time spent on the ventilator or at the study centre, or the total number who required endotracheal reintubation and further IPPV (table 2).

Four babies died after randomisation, two in each group. The cause of death was necrotising enterocolitis in two cases (both randomised to NCPAP) and severe periventricular leucomalacia, leading to withdrawal of intensive care in two cases (both randomised to headbox oxygen).

There was no significant difference between the groups in the incidence of chronic lung disease either at 28 days of age or at 36 weeks of corrected age. Likewise there were no differences in the incidence of cerebroventricular haemorrhage, periventricular leucomalacia (PVL), ROP, sepsis or feeding intolerance (table 2). Weight gain, both in the week following extubation and from extubation to discharge, was similar in both groups.

The first analysis performed after 50 patients showed no significant difference between the two groups using the O'Brien-Fleming

Table 3 Failure criteria

\begin{tabular}{lcc}
\hline & NCPAP $(n=16)$ & Headbox $(n=27)$ \\
\hline Apnoea & 12 & 10 \\
Increased $\mathrm{FIO}_{2}$ & 3 & $15(\mathrm{NNT}=4)$ \\
Respiratory acidosis & 1 & 2 \\
\hline
\end{tabular}

$\mathrm{NNT}=$ number needed to treat. criteria. ${ }^{12}$ However, enrolment was discontinued after the second interim analysis when the $p$ value was 0.013 for the primary outcome measure.

\section{Discussion}

Intermittent positive pressure ventilation has been a major advance in the care of preterm infants. It permits temporary support of pulmonary function and hence preservation of life. ${ }^{13}$ However, it is generally accepted that this form of ventilation places the infant at increased risk of pulmonary injury, leading to chronic lung disease. ${ }^{14}$ The presence of a foreign body in the trachea also provides a portal of entry for infection and may cause local damage to the upper airway. The optimal method of managing infants when they are judged no longer to need an endotracheal tube has been controversial. Theoretical reasons supporting the use of NCPAP in this role include prevention of atelectasis, recruitment of small airways, and decreased work of breathing. However, if extubation to headbox oxygen was shown to be equally effective, then this method of care would be favoured because it is less invasive.

We have found that extubation to a single nasal prong using a CPAP level of $7 \mathrm{~cm}$ water results in fewer infants having adverse clinical events, indicating the need for extra ventilatory support. The improvement in success rate with NCPAP is significant and clinically important. The stability provided by NCPAP in terms of reduced incidence of clinical failure criteria make it our preferred method of care. However, we recognise that the rescue treatment of babies failing a trial of headbox oxygen resulted in no difference in the need for endotracheal reintubation or in any other clinically important variables such as chronic lung disease or mortality. Others may view the results of this study differently, depending on their interpretation of the risks and benefits outlined.

Previous studies have shown conflicting results with respect to "successful extubation." The three smallest studies have shown that NCPAP is more effective, ${ }^{278}$ whereas the three largest have shown no difference between the two groups. ${ }^{5} 69$

Our study used the highest starting level of CPAP. Others have questioned the use of such levels, citing hypercapnia and hyperoxia as potential complications. ${ }^{6}$ We did not find an increased incidence of respiratory acidosis or of potential consequences of hyperoxia-ROP or CLD. The presence of an NCPAP tube, although shorter than an endotracheal tube, constitutes a foreign body in contact with delicate mucosa and therefore is a potential risk factor for infection. Previous studies have not reported on this important neonatal outcome. We found no increase in confirmed or suspected sepsis rates with the use of NCPAP. Positive airway pressure influences cerebral perfusion which in turn may contribute to haemorrhagic and ischaemic intracranial lesions. ${ }^{15}$ We found no significant difference in rates of either IVH or PVL. Gastric distension 
and feeding intolerance have been associated with the use of CPAP: there was no significant difference between groups with respect to incidence of episodes of feeding intolerance. Weight gain, both in the week after extubation and for the time from extubation until discharge, was similar in both groups. The benefits of NCPAP in providing stability in the week following extubation do not seem to be accompanied by clinically significant side effects.

1 Gregory GA, Kitterman JA, Phibbs RH, Tooley WH, Hamilton WK. Treatment of idiopathic respiratory distress syndrome with continuous positive airway pressure. $N$ Engl $\mathcal{F}$ Med 1971; 284:1333-40

2 Engelke SC, Roloff DW, Kuhns LR. Post-extubation nasal continuous positive airway pressure. Am $\mathcal{f}$ Dis Child 1982;136:359-61.

3 Andreasson B, Lindroth M, Svenningsen NW, Jonson B. Effects on respiration of CPAP immediately after extubation in the very preterm infant. Pediatr Pulmonol 1988;4:213-18

4 Field D, Vyas H, Milner AD, Hopkin IE. Continuous positive airway pressure via a single nasal catheter in preterm infants. Early Hum Dev 1985;11:275-80.

5 Annibale D, Hulsey T, Engstrom P, Wallin L, Ohning B. Randomised, controlled trial of nasopharyngeal continuous positive airways pressure in the extubation of very low birth weight infants. $\mathcal{F}$ Pediatr 1994;124:455-60.
6 Chan V, Greenough A. Randomised trial of methods of extubation in acute and chronic respiratory distress. Arch Dis Child 1993;68:570-2.

7 Higgins RD, Richter SE, Davis JM. Nasal continuous positive pressure facilitates extubation of very low birth weight neonates. Pediatrics 1991;88:999-1003.

8 So B-H, Tamura M, Mishina J, Watanabe T, Kamoshita S. Application of nasal continuous positive pressure to early extubation in very low birth weight infants. Arch Dis Child 1995;72: F191-3.

9 Tapia J, Bancalari A, Gonzalez A, Mercado M. Does continuous positive airways pressure (CPAP) during weaning from intermittent mandatory ventilation in very low birth weight infants have risks or benefits? A controlled trial. Pediatr Pulmonol 1995;19:269-79.

10 Papile LA, Burstein J, Burstein R, Koffler H. Incidence and evolution of subependymal and intra ventricular haemorrhage: A study of infants with birth weights less than 1500gm. F Pediatr 1978;92:529-34.

11 International Committee for the Classification of Retinopathy of Prematurity. An international classification of retinopathy of prematurity. Pediatrics 1984;74:127-33.

12 O'Brien PC, Fleming TR. A multiple testing procedure for clinical trials. Biometrics 1979;35:549-56.

13 Bancalari E, Sinclair JC. Mechanical ventilation. In: Sinclair JC, Bracken MB, eds. Effective care of the newborn infant. New York, NY: Oxford University Press Inc, infant. New

14 Korones SB. Complications. In: Goldsmith JP, Karotkin EH. Assisted ventilation of the neonate. Philadelphia, PA: WB Saunders, 1988:245-71.

15 Volpe JJ. Intra ventricular haemorrhage and brain injury in the premature infant. Clin Perinatal 1989;16:387-411. 